\title{
Proposta Metodológica para o Ensino de Química Utilizando o Tema Alimentação Saudável
}

\section{Methodological proposal for teaching chemistry using the healthy food theme}

\author{
Rogério Pacheco Rodrigues (rogeriopachecorp@hotmail.com) \\ Instituto Federal de Educação, Ciência e Tecnologia Goiano - Campus Morrinhos \\ Danyelle Stéphane Tavares Ferreira (dany.stephane91@ gmail.com) \\ Instituto Federal de Educação, Ciência e Tecnologia Goiano - Campus Morrinhos \\ Débora Augustinho da Silva (deborabio18@gmail.com) \\ Instituto Federal de Educação, Ciência e Tecnologia Goiano - Campus Morrinhos \\ Kelma Alves Mateus (kelmamateus@ gmail.com) \\ Instituto Federal de Educação, Ciência e Tecnologia Goiano - Campus Morrinhos \\ Lucilene Cândida dos Santos (lucilene_46@yahoo.com.br) \\ Instituto Federal de Educação, Ciência e Tecnologia Goiano - Campus Morrinhos \\ Cinthia Maria Felicio (cinthia.felicio@ifgoiano.edu.br) \\ Instituto Federal de Educação, Ciência e Tecnologia Goiano - Campus Morrinhos
}

\begin{abstract}
Resumo: Trata-se de uma proposta de ensino em que a contextualização e o trabalho multidisciplinar a partir da temática alimentação saudável pode servir como articuladora de relações entre diferentes áreas do conhecimento em ciências e matemática. Utilizando os três momentos pedagógicos de Delizoicov e levantamento de questões sócio científicas (QST), os alunos serão levados a refletirem sobre suas escolhas e pensarem alternativas para se buscar uma alimentação mais saudável. A oficina pedagógica pode ser desenvolvida em diferentes espaços pedagógicos seja em sala de aula ou laboratórios para ensino de ciências e várias estratégias didáticas são propostas, como a utilização de vídeos educativos, análise crítica de artigos científicos, sala de aula invertida e aulas experimentais. Desta forma, acreditamos que este trabalho possa ser de extrema importância para a área do ensino de ciências, pois apresentamos sugestões de como se pode trabalhar diferentes conteúdos de ensino com determinado tema de uma maneira interdisciplinar e em escala macroscópica por meio do desenvolvimento Da experimentação e protagonismo do aluno.
\end{abstract}

Palavras-chave: Multidisciplinaridade; Oficina pedagógica; Abordagens macroscópicas; Tomada de consciência.

Abstract: The goal of this work is presentend a teaching proposal for contextualization and multidisciplinary based on healthy eating and how it could be a good strategy to etablir relations between different areas of knowledge in science and mathematics. Using the three Delizoicov pedagogical moments and raising questions about Socioscientific Issues (SSI), the students will be able to reflect on their choices and think about alternatives to enjoy a healthier diet. The pedagogical workshop can be developed in different spaces as an ordinary classroam or in a chemistry laboratory and different teaching strategies are proposed, like the use of educational videos, critical analysis of scientific articles, flipped classroom and experimental classes. In this way, we believe that this work may be of extreme importance for the area of science education, as we present suggestions on how SE can work different contents of teaching 
with a specific subject in an interdisciplinary way and start with macroscopic aproach through the development of experimentation class and the student's protagonism.

Keywords: Multidisciplinary; Pedagogical workshop; Macroscopic approach; Take of consciousness.

\section{INTRODUÇÃO}

A temática de alimentos pode servir de contexto multidisciplinar para o ensino de ciências e matemática. Nesse sentido, foram planejadas as atividades que se seguem para o ensino das disciplinas de Biologia, Física, Química e Matemática como uma proposta didática que busca envolver os alunos e motivá-los ao estudo destas áreas. Além de ser um assunto motivador, a alimentação pode ser um tema rico conceitualmente, o que permite desenvolver conceitos químicos, físicos, biológicos, matemáticos entre outros, proporcionando aos estudantes compreender a importância deste estudo em suas vidas, de forma a conscientizá-los sobre a necessidade de uma dieta que esteja de acordo com suas necessidades diárias.

No entanto, esta proposta de Oficina Temática intitulada Alimentação Saudável: Produção, Contextualização e Interdisciplinaridade entre Ciências e a Matemática abrange situações do cotidiano e parte da reflexão dos alunos sobre seus hábitos alimentares e o que seria uma alimentação saudável para que assim, possam fazer suas escolhas de maneira consciente.

Sendo assim, os autores deste artigo optaram por trabalhar o conteúdo a partir de uma Oficina Temática seguindo os Parâmetros Curriculares Nacionais para o Ensino Médio (PCNEM) - área de Ciências da Natureza, Matemática e suas Tecnologias, que enfatiza a importância de se trabalhar a multidisciplinaridade e a contextualização dos conhecimentos no ensino das disciplinas que compõem essas áreas, na perspectiva de se pensar a organização curricular a partir de contextos, e não exclusivamente a partir de conceitos (BRASIL, 1999).

De acordo com Silva e Tavares (2005) a multidisciplinaridade trata-se de estudos relacionados a informações de várias disciplinas para estudar um determinado elemento, estabelecendo uma integração entre estas disciplinas. Ou seja, o ensino multidisciplinar traz contribuições significativas a uma disciplina específica, porque "ultrapassa as 
disciplinas, mas sua finalidade continua inscrita na estrutura da pesquisa disciplinar" (NICOLESCU, 2000. p. 14).

No entanto, no material intitulado Oficinas Temáticas no Ensino Público: Formação Continuada de Professores no Estado de São Paulo, pode ser observado nas discussões que as oficinas temáticas baseadas no pressuposto da contextualização, além de apresentarem elevado grau de importância para a sociedade, o que já justificaria sua discussão, ainda podem favorecer o desencadeamento de diversos conhecimentos. Os conteúdos envolvidos e conhecimentos discutidos podem ser apresentados por meio de experimentos, com intuito de fornecer subsídios para que os alunos possam entender o tema de estudo e se posicionarem perante as questões que este pode trazer para o debate na sociedade (SÃO PAULO, 2007).

No contexto do ensino de química, Coelho e Marques (2007) salientam que o uso de Temas Químico-Sociais (TQS) no processo de ensino se torna uma ferramenta potente para inúmeras tarefas, pois os temas são extraídos das relações do sujeito com seu contexto (local, regional, nacional ou mundial) e seu desenvolvimento viabiliza a problematização dos conteúdos ministrados.

Sendo assim, este artigo tem como objetivo apresentar uma proposta didática para o ensino interdisciplinar entre conteúdos de Biologia, Química e Matemática, utilizando como estratégia de ensino a Oficina Temática.

\section{CONTEXTO METODOLÓGICO DA PROPOSTA}

Esta atividade pedagógica partiu da orientação dos Professores e da Coordenadora do Curso de Pós-Graduação em Ensino de Ciências e Matemática do IF Goiano de Morrinhos, como atividade avaliativa da disciplina de Tópicos Especiais, a qual solicitou aos estudantes que se organizassem em grupos com diferentes formações para trabalhar a Temática Alimentos envolvendo o contexto interdisciplinar.

Assim, foi planejada a realização de uma Oficina Temática que teria como objetivo principal propiciar o desenvolvimento e a aprendizagem de conteúdos que pudessem auxiliar na vida dos alunos e ainda contribuir para entendimento de conceitos estudados em Biologia, Química e Matemática como disciplinas de fundamental 
importância para o entendimento de conceitos científicos, a partir da temática Alimentação Saudável.

Os conteúdos delimitados para serem explorados durante a execução desta proposta são: função e importância dos Alimentos, alimentação saudável, ecologia de Populações e comunidades, sistema digestório e efeitos fisiológicos causados pela má alimentação, sendo estes para o ensino da Biologia. Para o ensino da química, elencamos as análises Qualitativa e Quantitativa, termoquímica, cadeias carbônicas e suas respectivas classificações, funções orgânicas; biomoléculas e suas propriedades: carboidratos, proteínas, lipídeos, enzimas, vitaminas, sais minerais. A estatística, matemática financeira, funções, cálculo do IMC, operações matemáticas, foram os conteúdos selecionados para a disciplina de matemática.

\section{O Desenvolvimento das Aulas:}

Para a organização da oficina, a mesma será estruturada nos Três Momentos Pedagógicos propostos por Delizoicov, Angotti e Pernambuco (2011), que são: Problematização Inicial (PI), Organização do Conhecimento (OC) e Aplicação do Conhecimento (AC) seguindo uma abordagem Ciência, Tecnologia, Sociedade e Ambiente (CTSA) pautada nos conteúdos do Currículo de Referência da Rede Estadual de Educação do Estado de Goiás, podendo assim realizar algumas adaptações curriculares.

\section{Primeiro Momento Pedagógico: Problematização Inicial:}

Para a problematização inicial, primeiramente, poderá ser solicitado a cada estudante que responda a um questionário e expresse individualmente sua opinião em relação às questões, como: O que você sabe sobre os alimentos? Cite alguns alimentos que você considera como saudáveis? Por que? Por que nos alimentamos? Você tem uma alimentação saudável? Na sua residência a sobra de comida é depositado onde? Em sua opinião, o que são os aminoácidos e proteínas?

Em seguida, os participantes poderão resolver alguns exercícios de Química Orgânica, com o propósito de identificar as funções orgânicas nas fórmulas estruturais de alguns compostos presentes na composição química de alimentos e chás, utilizando 
trabalhos como de Pazinato e Braibante (2013), Braibante et al., (2014) e Silva et al., (2017).

Posteriormente, todas as questões deverão ser discutidas oralmente com os alunos e os professores de Química e Biologia, os quais poderão realizar anotações relevantes no quadro. Durante a discussão, os docentes podem fazer uso do artigo intitulado "Análise Qualitativa de Proteínas em Alimentos por meio de Reação de Complexação do Íon Cúprico" de Almeida et al., (2012) e o material de Pinheiro (2005), conceituando os temas como o funcionamento do sistema digestório, o controle da ação digestiva, a importância dos alimentos e de uma alimentação saudável, carboidratos, proteínas, lipídios, vitaminas, sais minerais, a função dos alimentos, o consumo de frutas e legumes, consumo excessivo de determinados alimentos, bem como os efeitos fisiológicos causados pela má alimentação, doenças com restrições alimentares e informações em rótulos etc.

\section{Segundo Momento Pedagógico: Organização do Conhecimento:}

Nesta etapa os alunos realizarão o experimento investigativo para determinação de Proteína em alguns dos alimentos que consumidos no dia a dia dos estudantes, seguindo a metodologia de Almeida et al., (2012). Assim, algumas análises relacionadas ao controle de qualidade poderão ser desenvolvidas e posteriormente, discutir sobre a interpretação das informações contidas em rótulos.

Em seguida pode ser exibido o Filme "Consumed - What are you Eating? que retrata a história de uma mãe que começa a investigar os problemas misteriosos de saúde do seu filho e acaba chegando até a indústria dos alimentos geneticamente modificados.

Aproveitando a ideia geral do filme, o professor pode explanar algumas metodologias analíticas para a determinação de Agrotóxicos em vegetais, enfatizando neste estudo a cenoura (ARAÚJO et al., 2016). Sendo assim, poderia ser apresentado os dados do último Relatório das Análises de Amostras Monitoradas no Período de 2013 a 2015 pelo Programa de Análise de Resíduos de Agrotóxicos em Alimentos (PARA) da Agência de Vigilância Sanitária (ANVISA). 
Para trabalhar os conteúdos de matemática financeira e funções matemáticas, o docente utilizará como recurso os valores gastos para a produção de alimentos, que podem ser consumidos no dia a dia de seus alunos, contextualizando com uma simulação de uma quantidade de pessoas que serão servidos em determinada reunião, a partir da produção destes alimentos e bebidas.

\section{Terceiro momento pedagógico: aplicação do conhecimento:}

Para a aplicação do conhecimento, em relação aos conteúdos estudados durante a oficina, o docente pode optar por três atividades para verificação da aprendizagem. Inicialmente, levantar algumas questões a respeito da classificação dos animais, como por exemplo, o frango como ave ou o porco como suíno e/ou os diferentes tipos de vegetais, os alunos serão organizados em grupos, para realizarem os estudos e apresentarem para toda turma.

Levando em consideração a importância de estudar o conteúdo energético dos alimentos, os alunos irão efetuar o cálculo do IMC (Índice de Massa Corpórea) reconhecido pela OMS (Organização Mundial da Saúde) como a principal referência para classificação das diferentes faixas de peso. O cálculo do IMC será feito usando a fórmula matemática: (Peso $\div$ altura $\mathrm{x}$ altura), onde serão destacadas as faixas abaixo do peso que varia de 17 a 18,4 kg/m², peso normal varia entre 18,5 a $24,9 \mathrm{~kg} / \mathrm{m}^{2}$, acima do peso 25 a $29,9 \mathrm{~kg} / \mathrm{m}^{2}$ e obesos 30 a $34,9 \mathrm{~kg} / \mathrm{m}^{2}$. Cada aluno será avaliado e verificado qual estágio está, buscando alertar e enfatizar a importância de uma vida saudável para o desenvolvimento da qualidade de vida. Em seguida os mesmos deverão calcular as calorias e o conteúdo energético dos alimentos: Pão Integral com Gergelim, Patê de Frango com Cenoura, Suco de Uva Integral e Chá de Gengibre com Canela utilizando a Tabela Brasileira de Composição de Alimentos (TACO).

Após estes cálculos realizaremos uma análise e reflexão, utilizando os resultados obtidos dos valores de IMC calculados, e discutiremos se a alimentação dos mesmos está sendo saudável de acordo com os alimentos escolhidos. Para conscientizá-los sobre a importância da prática de exercícios físicos, os alunos poderão calcular, por meio de tabelas, quanto tempo eles precisam se exercitar, em uma modalidade específica (corrida, natação, futebol etc.), para consumir o valor energético dos alimentos 
escolhidos. Visto que, além de abordar conceitos de termoquímica, também envolve a utilização de matemática básica na realização dos cálculos necessários.

Com base na atividade experimental da Maionese, os alunos participarão de um Quizz, em que deverão responder alguns Mitos ou Verdades sobre o produto. Para esta atividade, serão confeccionadas plaquinhas para identificar a opinião dos alunos. Posteriormente os dados obtidos, serão analisados e comparados com a Resolução RDC $n^{\circ} 276$, de 22/09/2005 (BRASIL, 2005), utilizando a estatística e discutidos com os integrantes da oficina.

Neste estudo utilizará como método avaliativo, a avaliação formativa com o objetivo de acompanhar a aprendizagem dos alunos e auxiliar na tomada de decisões dos professores e auxiliar os estudantes a melhorarem seu desempenho no estudo da temática proposta.

Assim, em todas as etapas da oficina será necessário avaliar o aluno, ou seja, será processual. Pelo próprio caráter processual podemos também dizer que esta avaliação é dinâmica. A dinamicidade permite que ao tomar as decisões pedagógicas o professor auxilie o aluno a alcançar um novo estado de qualidade, que seja melhor e mais satisfatório e ao mesmo tempo possa reformular o ensino quantas vezes se fizerem necessárias. A avaliação em processo, ou seja, a avaliação formativa, prevê a inclusão dos alunos para assumirem com o professor os riscos das decisões tomadas, pois a parceria entre os professores e alunos pode constituir um pacto pelas finalidades da aprendizagem de maneira coletiva.

\section{CONSIDERAÇÕES FINAIS}

A utilização de temáticas envolvendo alimentação, possui um amplo potencial pedagógico pela contextualização multidisciplinar, permitindo explorar diferentes contextos, seja em ciências e matemática, possibilitando que os professores possam trabalhar em parceria com outras áreas do conhecimento.

Os três momentos pedagógicos propostos podem permitir a exploração do tema de forma instigadora e motivar os estudantes para o estudo e discussão da temática em diferentes realidades e perspectivas, possibilitando debates e reflexões sobre a importância do conhecimento científico na tomada de decisões e escolhas para uma 
alimentação com maior qualidade nutricional.

\section{REFERÊNCIAS}

ALMEIDA, V. V.; CANESIN, E. A.; SUZUKI, R. M.; PALIOTO, G. F. Análise Qualitativa de Proteínas em Alimentos por meio de Reação de Complexação do Íon Cúprico. Química Nova na Escola, v. 35, n. 1, p. 34-40, 2013.

ARAÚJO, E. A.; LARA, M. C. R.; REIS, M. R.; VIRIATO, R. L. S.; ROCHA, R. A. R.; GONÇALVES, R. G. L.; HELENO, F. F.; QUEIROZ, M. E. L. R.; TRONTO, J.; PINTO, F. G. Determination of Haloxyfop-Methyl, Linuron, and Procymidone Pesticides in Carrot Using SLE-LTP Extraction and GC-MS. Food analytical methods, v.9, n. 5, p. 1344-1352, 2016.

BRAIBANTE, M. E. F.; SILVA, D.; BRAIBANTE, H. T. S.; PAZINATO, M. S. A Química dos Chás. Química Nova na Escola, v. 36, n. 3, p. 168-175, 2014.

BRASIL. AGÊNCIA NACIONAL DE VIGILÂNCIA SANITÁRIA. Resolução RDC $n^{\circ}$ 276, de 22/09/2005. Regulamento Técnico para especiarias, temperos e molhos. Diário Oficial da União; Poder Executivo, de 23 de setembro de 2005.

BRASIL. Secretaria de Educação Média e Tecnológica. Parâmetros Curriculares Nacionais: Ensino Médio: Ciências da Natureza, Matemática e suas Tecnologias. Brasília: MEC/SEMTEC, v. 3, 1999.

COELHO, J. C.; MARQUES, C. A. Contribuições freireanas para a contextualização no ensino de Química. Ensaio Pesquisa em Educação em Ciências, v. 9, n. 1, 2007.

CURRÍCULO DE REFERÊNCIA DA REDE ESTADUAL DE EDUCAÇÃO DE GOIÁS. Disponível em: <

http://www.seduc.go.gov.br/imprensa/documentos/arquivos.pdf $>$ acesso em 10 de maio de 2019.

DELIZOICOV, D.; ANGOTTI, J. A.; PERNAMBUCO, M. M. Ensino de Ciências: fundamentos e métodos. 4. ed. São Paulo: Cortez, 2011.

NICOLESCU, B. et al. Educação e Transdisciplinaridade. Brasília: UNESCO, 2000 (Edições UNESCO).

PAZINATO, M. S.; BRAIBANTE, M. E. F. Oficina Temática Composição Química dos Alimentos: Uma Possibilidade para o Ensino de Química. Química Nova na Escola, v. 36, n. 4, p. 289-296, 2014.

PINHEIRO, D. M. A Química dos alimentos: carboidratos, lipídios, proteínas e minerais. Maceió: EDUFAL, 2005. 
SÃO PAULO. Secretaria da Educação. Oficinas temáticas no ensino público:

formação continuada de professores. Secretaria da Educação, Coordenadoria de Estudos e Normas Pedagógicas; Organização de Dayse Pereira da Silva; coordenação de Maria Eunice Ribeiro Marcondes. - São Paulo: FDE, 2007. 108 p.

SILVA, F. E. F.; RIBEIRO, V. G. P.; GRAMOSA, N. V.; MAZZETTO, S. Temática Chás: Uma Contribuição para o Ensino de Nomenclatura dos Compostos Orgânicos. Química Nova na Escola, v. 39, n. 4, p. 329-338, 2017.

SILVA, I. B.; TAVARES, O. A. O. Uma Pedagogia Multidisciplinar, Interdisciplinar ou Transdisciplinar para o Ensino/Aprendizagem da Física. Holos, v. 21, 2005. 